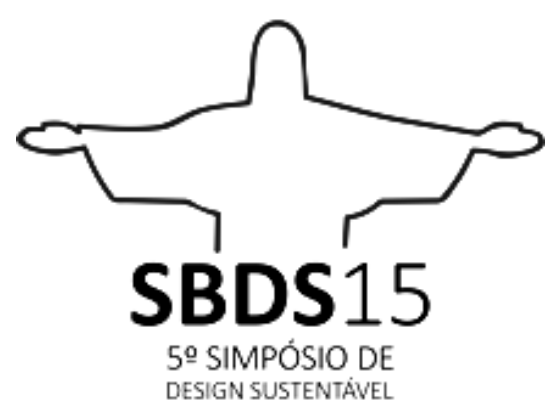

Rio de Janeiro, 11 de novembro a 13 de novembro de 2015

\title{
A pesquisa voltada para o estudo de materiais e produtos sustentáveis: relatos e experiências de grupos de pesquisa na Gráfica da Universidade Federal do Rio Grande do Sul
}

\author{
Jussara Smidt Porto \\ Universidade Federal do Rio Grande do Sul \\ jussara.porto@ufrgs.br \\ Eliana Paula Calegari \\ Universidade Federal do Rio Grande do Sul \\ elianapaulac@gmail.com \\ [Mariana Piccoli \\ Universidade Federal de Santa Maria \\ marianap.piccoli@gmail.com] \\ [Alice de Oliveira Meditsch \\ Universidade Federal do Rio Grande do Sul \\ alicemeditsch@gmail.com] \\ [Clarissa Coussirat Angrizani \\ Universidade Federal do Rio Grande do Sul \\ cangrizani@hotmail.com]
}

\begin{abstract}
Resumo: A pesquisa, com o intuito de gerar produtos sustentáveis, é uma realidade que deve ser vista como imprescindível nos tempos atuais. Não se pode negar que, cada vez mais, necessita-se de produtos para o nosso dia a dia, mas constata-se que não há uma preocupação mais efetiva com o ciclo de vida dos novos produtos, com os materiais de que eles são feitos e com o seu descarte. Este artigo pretende expor as ações dos grupos de pesquisa no Núcleo de Desenvolvimento de Projetos em Papel (NDePP) da Gráfica da Universidade Federal do Rio Grande do Sul (UFRGS), no sentido de promover o desenvolvimento de novos produtos que estejam de fato comprometidos com a temática da sustentabilidade. A partir da criação do Núcleo, implantaram-se os grupos de pesquisas que já produziram e estão produzindo estudos para o desenvolvimento de produtos focados em ecodesign. Este estudo relata as pesquisas sobre tingimento natural de papel machê, processos de impermeabilização, desenvolvimento de produtos a partir do resíduo de papel e pesquisa com compósitos. Assim, a partir do relato destas experiências, este artigo busca estimular e divulgar o trabalho feito por estes grupos de pesquisa para que, cada vez mais, se promovam
\end{abstract}


estudos multidisciplinares nas instituições de ensino nas quais se buscam sustentabilidade e inovação.

Palavras-chave: estudo de materiais, produtos sustentáveis, resíduos de papel.

Abstract: The research, in order to generate sustainable products, is a reality that must be seen as essential in actual times. Can not be denied that an increasingly need of products for our everyday life, it appears that there is a more effective concern about the life cycle of new products, the materials from which they are made and discarding. This article aims to demonstrate the actions of the research groups that are being made in the "Núcleo de Desenvolvimento de Projetos em Papel/NDePP (Center of Development of Projects on Paper) of printing industrie of the Federal University of Rio Grande do Sul (UFRGS), to promote the development of new products that are, in fact, committed to the theme of sustainability. From the creation of the Center, the research groups have produced and are producing studies to develop products focused on ecodesign. This study reports research on natural dyeing on paper-mache, waterproofing processes, product development from paper lint and research with composites. Thus, from the account of these experiences, this article seeks to stimulate and disseminate the work done by these research groups so that, increasingly, to promote multidisciplinary studies in educational institutions in which they seek sustainability and innovation.

Keywords: material's study, sustainable products, paper waste

\section{INTRODUÇÃO}

A humanidade se reconstrói e se lança para novos significados e novos horizontes, nos quais os aspectos ambientais, sociais e econômicos se fundem para a construção de um novo paradigma. Essa reconstrução tem gerado atitudes por parte da sociedade que sinaliza um maior comprometimento em relação à sustentabilidade.

Segundo Kazazian (2005), vivemos uma idade que se impõe o desenvolvimento sustentável, estando ao nosso alcance imaginar soluções que favoreçam a prosperidade sem que isso implique regredir em qualquer setor dos sistemas econômicos ou natural. Neste contexto, segundo Calegari (2013) as preocupações com as questões ecológicas têm gerado interesse na utilização de fontes renováveis, sustentáveis e de materiais que podem ser compostados.

As organizações de todos os tipos preocupam-se cada vez mais em atingir e demonstrar um desempenho ambiental correto, controlando o impacto de suas atividades, de seus produtos e serviços considerando suas políticas e seus objetivos ambientais (CENTRO SEBRAE DE SUSTENTABILIDADE, 2013). Devido a isto, o desenvolvimento de projetos sustentáveis pelas empresas tem aumentado ao longo dos últimos anos, gerando também para as instituições de ensino, uma crescente demanda e expansão no desenvolvimento da pesquisa nesta área.

Nesta virada de milênio, não é possível ficar indiferente às exigências da sociedade e de um mercado em crescente processo de conscientização ecológica. De acordo com Wu e Liao (2012), "nos dias atuais, estão sendo desenvolvidos materiais de fontes renováveis e biodegradáveis como resultado da preocupação ambiental". Neste contexto, e atendendo ao Plano de Desenvolvimento Institucional (PDI) da instituição, a da Universidade Federal do Rio Grande do Sul (UFRGS) desenvolve e estimula programas de sustentabilidade que atendam às exigências tanto de âmbito ambiental, social e econômico. Assim, com o intuito de contribuir para a transformação social e atender aos novos objetivos e demandas exigidas por parte do Governo Federal, a Gráfica UFRGS vem desenvolvendo ações que vão ao encontro destes propósitos. Conforme Curtis (2011), diante da natureza de sua atividade, a redução de resíduos e preservação do ambiente são aspectos que demandam atenção. Com efeito, a questão ambiental é um fator determinante no contexto da produção gráfica.

Em 2010, a fim de reutilizar os resíduos de papel gerados pela Gráfica, foi criado o Núcleo de Desenvolvimento de Projetos em Papel (NDePP) que tem como objetivo, através dos grupos de pesquisa, 
realizar estudos para promover o reaproveitamento do resíduo de papel, pesquisar técnicas para o desenvolvimento de produtos e capacitar comunidades para que produzam e comercializem os artefatos desenvolvidos. Sendo assim, o objetivo deste trabalho é relatar os estudos sobre técnicas, materiais e produtos sustentáveis que foram pesquisados no NDePP, através dos grupos de pesquisas que foram criados ao longo dos anos, e enfatizar a importância da implantação destes grupos para a viabilidade destas pesquisas.

\subsection{Gráfica da UFRGS e o Núcleo de Desenvolvimento de Projetos em Papel (NDePP)}

A Gráfica da Universidade Federal do Rio Grande do Sul está situada no Campus da Saúde, na Rua Ramiro Barcelos, 2500, em Porto Alegre. A sua atividade fim é imprimir a produção gráfica da comunidade acadêmica. Em relação a grande quantidade de passivo gerado pela Gráfica, fez com que o processo de conscientização ecológica se concentrasse na criação, em 2007, do Laboratório de Reciclagem de Papel, cujo objetivo foi à produção de papel artesanal a partir das aparas (resíduos). Instalado nas dependências da própria Gráfica, o laboratório foi equipado com os materiais necessários e iniciou a produção de papel reciclado artesanal em diferentes formatos, gramaturas e cores.

Em 2008, iniciou-se o uso da técnica de papel machê na confecção de diversos tipos de objetos. Em 2010 o Laboratório transformou-se no Núcleo de Desenvolvimento de Projetos em Papel (NDePP), ampliando seus objetivos para o desenvolvimento de produtos a partir de técnicas com papel, sempre utilizando as aparas da produção interna.

O foco atual do NDePP é estudar as possibilidades de trabalho com o resíduo de papel, criando produtos com o objetivo final de capacitar ONGs, associações de artesanato e cooperativas no sentido de fortalecerem-se como associação e gerarem renda para seus colaboradores. Desta forma, volta-se para a efetivação do papel social da Gráfica, ao buscar uma interação com as comunidades externas e promover o empoderamento das pessoas envolvidas. No sentido de atender esta demanda, e percebendo a complexidade da construção deste conhecimento, procurou-se estimular a formação de grupos de pesquisa para promover um diálogo com outras áreas e alcançar a interdisciplinaridade a fim de possibilitar novos paradigmas para a investigação.

Sendo assim, o Núcleo apresenta-se como um espaço de experimentação de grupos de pesquisas (Figura 1), onde são testados possíveis caminhos e soluções, e realizados diversos tipos de pesquisa. Devese salientar que o pensamento ecológico é a diretriz do trabalho, determinando decisões com relação a materiais e processos empregados. Através do NDePP já se constituíram vários grupos de pesquisa que promoveram a evolução dos estudos relacionados com o desenvolvimento da linha adotada. Estes grupos foram formados por estudantes dos cursos de Design, Artes Plásticas, Engenharia, Geografia e Técnico Ambiental.

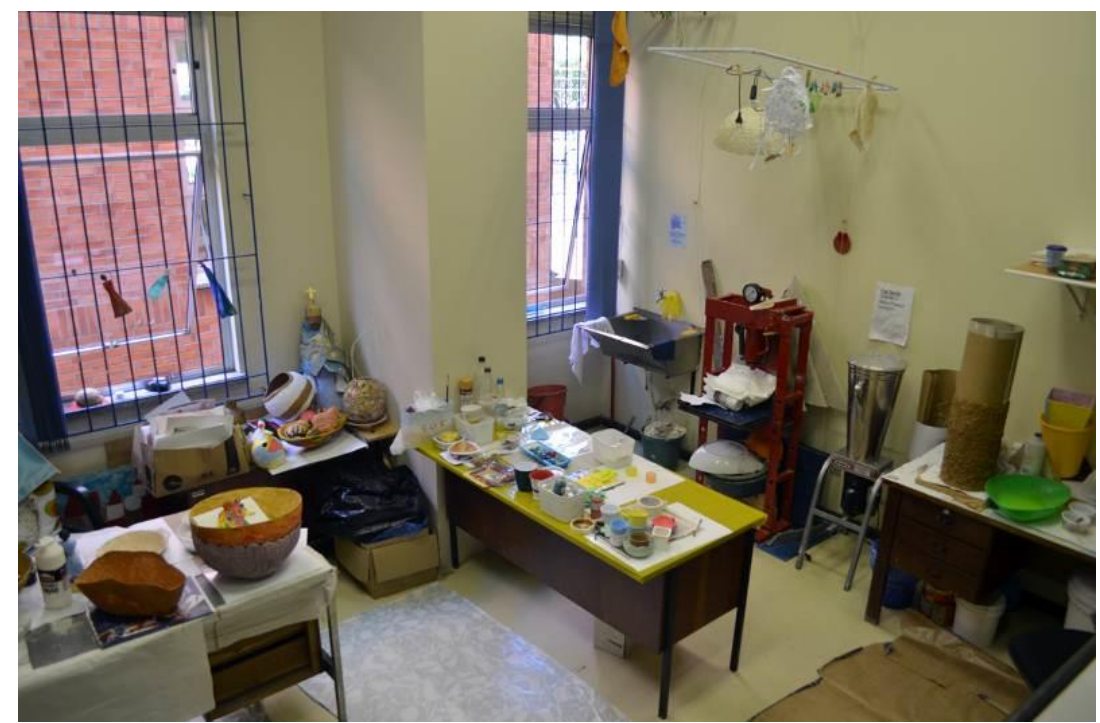

Figura 1 - Dependências do NDePP, situado internamente à Gráfica da UFRGS.

Fonte: Elaborado pelos autores, com base na pesquisa realizada. 


\section{DESENVOLVIMENTO}

Os procedimentos metodológicos caracterizam-se como uma pesquisa descritiva, pois "os fatos são observados, registrados, analisados, classificados e interpretados, sem que o pesquisador interfira sobre eles" (PRODANOV; FREITAS, 2009, p. 63). Assume a forma de estudo de campo, já que os dados são coletados (tais como ocorrem, espontaneamente) diretamente no local em que se dá o fato estudado, utilizando muito mais técnicas de observação do que de interrogação.

Dentre os procedimentos técnicos, apresenta-se a pesquisa bibliográfica, já que no referencial teórico sobre práticas de responsabilidade socioambiental buscaram-se informações em livros, periódicos e trabalhos acadêmicos pertinentes sobre o assunto. Também a pesquisa documental, pois se investigaram relatórios fornecidos por empresas e unidades parceiras ou relatórios semestrais internos da Gráfica, artigos publicados sobre as técnicas pesquisas. A coleta de dados ocorreu por meio dos seguintes instrumentos: observação e acompanhamento in loco dos trabalhos desenvolvidos, utilizando-se também registro fotográfico.

\subsection{Práticas de pesquisa desenvolvidas pelos grupos de pesquisa do NDePP}

Os grupos de pesquisa podem se tornar o diferencial para a pesquisa das instituições e para a formação do aluno, gerando uma formação diferenciada e possibilitando uma educação complementar de qualidade. No contexto do Núcleo da Gráfica, é importante ressaltar a evolução ocorrida no NDePP, através da criação destes grupos. Eles contribuíram para a reflexão e questionamentos na busca de novos conhecimentos.

Pode-se exemplificar com a sequência das pesquisas que foram surgindo neste laboratório. Através do objetivo inicial do NDePP, que tinha como foco a criação de produtos com materiais biodegradáveis e sustentáveis, surgiu a primeira proposta que foi pesquisar o desenvolvimento de produtos a partir da técnica de papel machê. Este processo demonstrou que o produto ficava muito vulnerável em relação à umidade, o que limita a sua aplicação, necessitando novas pesquisas, e, a partir disso, foram buscados novos caminhos.

Primeiramente, foi realizada a pesquisa sobre o tingimento natural do papel machê, em que se obteve bom resultado, pois se conseguiu uma coloração natural aos produtos. Depois disso, buscou-se a impermeabilização no papel machê com materiais à base de água, pesquisa na qual se obteve um bom resultado na medida em que o material impermeabilizante não agride a natureza.

Apesar destes resultados positivos, se constatou que havia necessidade de buscar um processo de desenvolvimento do produto que fosse mais viável sob o ponto de vista da execução, pois a confecção dos produtos de papel machê eram muito vulneráveis em relação à moldagem e secagem. Devido a essa demanda, a pesquisa evoluiu para o desenvolvimento de um compósito a partir da mistura de uma resina com o resíduo de papel, que ainda está sendo desenvolvida. A seguir, serão descritos os processos de desenvolvimento das pesquisas citadas anteriormente.

\subsection{Desenvolvimentos de produtos a partir do resíduo de papel gerados na Gráfica, em uma comunidade de Porto Alegre.}

Objetivando estender o papel social do NDePP e investigar a possibilidade de se desenvolver produtos a partir de resíduos gerados na Gráfica da UFRGS, a diretora da Gráfica, Jussara Porto desenvolveu sua dissertação intitulada "Avaliação do uso da metodologia Human Centered Design (HCD) para qualificação de técnicas de produção de artefatos a partir de resíduos de papel em comunidade de Porto Alegre/RS", no mestrado em Design no Centro Universitário Ritter dos Reis, em janeiro de 2013. A dissertação é o relato da experiência das oficinas oferecidas pelo NDePP a um grupo de artesãs que frequentam o Núcleo Comunitário e Cultural de Belém Novo (NCCBN), que promoveu a experiência da criação de produtos em papel machê.

Neste estudo, buscou-se aplicar uma metodologia que possibilitasse fazer o diagnóstico da realidade atual e identificasse as necessidades desta comunidade. Por meio da metodologia HCD, buscouse conhecer os desejos das participantes, e partindo das suas expectativas, criaram-se produtos para datas comemorativas e iniciou-se a orientação para formação de uma cooperativa. Segundo Memória $(2005$,$) os$ princípios básicos de metodologia são importantes para qualquer projeto de design. Assim, com a aplicação 
desta metodologia, foi projetado todo o processo da intervenção na comunidade e se constituíram as ferramentas pela qual foram determinadas as diretrizes desta pesquisa.

As oficinas foram ministradas pelos integrantes do grupo de pesquisa existente no NDePP daquele período, o funcionário Paulo Cesar Rocha e a estudante de Design Alice Meditsch. Durante os doze encontros, as participantes aprenderam técnicas de reutilização do resíduo de papel (papietagem e papel machê). A partir dessas técnicas, foram definidos os produtos que seriam modelados, e realizadas as demais etapas da produção.

Através desta experiência constata-se que esta metodologia fornece ferramentas que tornam a implantação do projeto bastante estimulante e viável. Apesar de não se ter chegado à plenitude do desafio estratégico definido nos primeiros encontros com as artesãs, conseguiu-se delinear possibilidades que poderão ajudar na realização dos desejos das pessoas envolvidas, tanto das artesãs que participaram das oficinas, quanto dos facilitadores do NDePP (Figura 2).

Esta pesquisa se constituiu como um plano piloto para o desenvolvimento de novas frentes, fornecendo subsídios para a implantação de projetos com outros cenários. Constatou-se a necessidade da percepção mais fortalecida do designer como um agente de intervenção, da necessidade de novos integrantes para novas pesquisas com novos materiais.
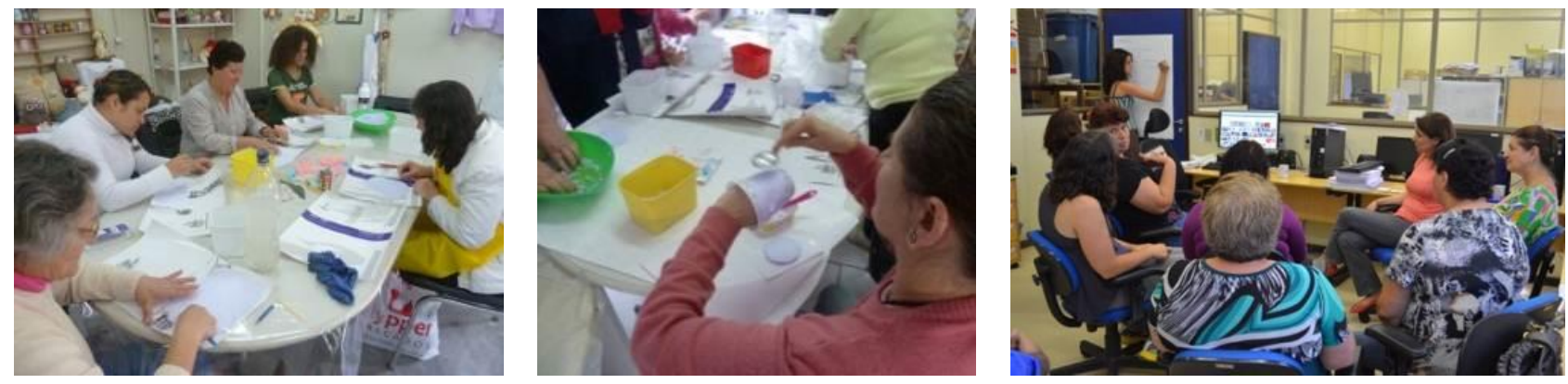

Figura 2 - Atividades realizadas durante os encontros: experimentações na massa de papel, modelagem de artefatos e conversa sobre as expectativas.

Fonte: Elaborado pelos autores, com base na pesquisa realizada.

\subsection{Pesquisa sobre o tingimento natural do papel}

Seguindo o propósito de se trabalhar com materiais naturais, o grupo de pesquisa constituído pela então mestranda em Design Mariana Piccoli e a acadêmica do curso de Design, Alice Meditsch iniciou a pesquisa sobre o tingimento do papel machê com materiais naturais. Os corantes eleitos foram aqueles que tiveram melhor resultado em termos de coloração e fixação na bibliografia pesquisada, além de serem facilmente adquiridos na região Sul do Brasil. O papel machê foi produzido a partir das aparas de papel provindas da Gráfica da Universidade Federal do Rio Grande do Sul. As formas utilizadas para moldá-lo foram selecionadas a partir de estudos prévios realizados no NDePP. O pote de uma marca regional de iogurte mostrou-se a forma apropriada, pois facilita a modelagem e desmoldagem, além do seu apelo estético (PICCOLI et al., 2014).

Selecionaram-se os seguintes corantes para o tingimento: açafrão, beterraba, café, canela, casca de cebola, casca de nozes, colorau, erva-mate, macela e pinhão. A obtenção do corante foi realizada por meio da fervura do material por 30 minutos. Após os 30 minutos de fervura, a água colorida era coada, misturando-se uma colher de sopa de sal (mordente, para fixação da cor). Juntou-se a massa de papel, que já havia sido preparada anteriormente, com a água tingida, ainda quente. 0 papel ficou de molho por 48 horas, sendo que a cada 12 horas mexia-se a mistura para garantir que o corante penetrasse no papel de forma homogênea.

Após este processo, o papel era peneirado, torcido, misturado com cola caseira e modelado sobre uma forma plástica (Figura 3). Como resultado observou-se que o papel foi tingido e, apesar da cor da massa, após a secagem, ter se modificado e conferido um aspecto mais desbotado, responde bem aos corantes naturais, sendo uma possibilidade a mais de experimentações de materiais, podendo-se evitar assim o uso de tintas e corantes tóxicos. 

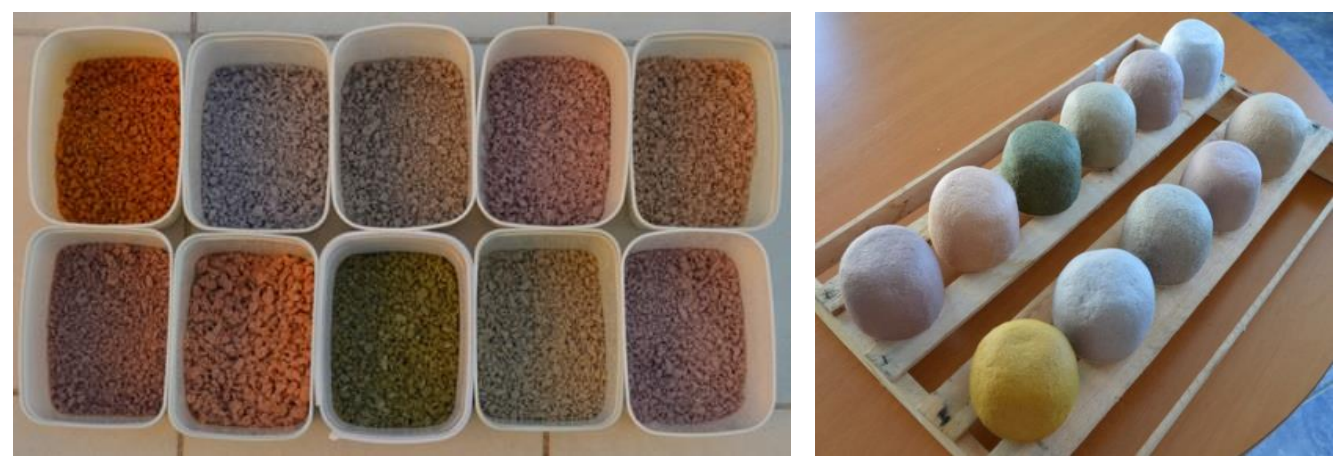

Figura 3 - Papel tingido, já peneirado e torcido (esquerda) e potes modelados, no processo de secagem (direita).

Fonte: Elaborado pelos autores, com base na pesquisa realizada.

\subsection{Pesquisa sobre impermeabilização do papel}

Outra pesquisa realizada pelo Núcleo de Desenvolvimento de Projetos em Papel refere-se à impermeabilização dos produtos feitos de papel reciclado. Uma vez impermeabilizados, os artefatos poderão ser utilizados para uma gama de aplicações muito maior do que podem atualmente, com a condição de fragilidade à água.

O balizador da pesquisa foi à utilização de materiais biodegradáveis e não tóxicos no recobrimento. Assim, com base em pesquisa bibliográfica e em experimentações preliminares, foram testados os seguintes produtos: cola branca, verniz acrílico, goma laca incolor, termolina e verniz marítimo a base de água. Foram feitas amostras de papel machê que receberam nenhuma, uma, duas ou três demãos dos produtos, e posteriormente foram testadas em relação à absorção de água em função do tempo.

Além disso, variou-se a própria resina utilizada na confecção das peças em papel machê. Foram feitas amostras utilizando cola branca, cola caseira (à base de farinha) e cola de celulose (CMC). $O$ ensaio deu-se da seguinte forma: as amostras eram pesadas secas, e depois imersas em água, e pesadas em intervalos de tempo pré-determinados: 10 e 30 segundos, 1 minuto, 5 minutos e 10 minutos. Em cada pesagem, observava-se também o aspecto físico e de amolecimento do material.

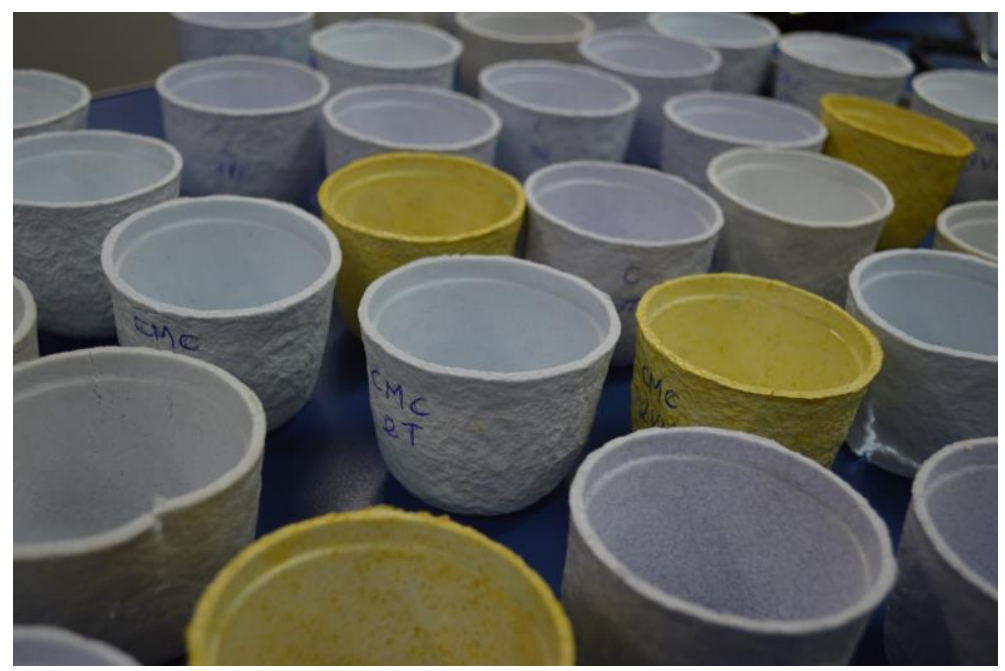

Figura 4 - Amostras confeccionadas para os testes de impermeabilização.

Fonte: Elaborado pelos autores, com base na pesquisa realizada.

Os resultados desta pesquisa apontam que o material que proporcionou a melhor impermeabilização foi o verniz marítimo à base de água, com pouca diferença de dados na aplicação em duas ou três camadas. Por exemplo: na massa confeccionada com cola branca e recoberta com verniz marítimo em duas demãos, a pesagem final, após 10 minutos de imersão em água, apresentou uma média 
de $101,8 \%$ em relação à massa inicial (100\%). Ou seja, a taxa de absorção foi somente $1,8 \%$. A massa de cola caseira é o material mais frágil; mesmo recoberto com verniz marítimo, o mais eficaz dos recobrimentos, o resultado foi insatisfatório; não foi possível realizar a pesagem aos 10 minutos, pois a amostra se desmanchava antes desse tempo.

Esta pesquisa mostra que o papel machê pode passar por um processo de impermeabilização com materiais menos tóxicos e não agressivos ao meio ambiente, de maneira que seu uso alcance uma gama maior de aplicações.

\subsection{Pesquisa com biocompósito utilizando o papel como reforço e uma resina biodegradável como matriz}

A pesquisa sobre compósitos na Gráfica da Universidade Federal do Rio Grande do Sul surgiu pelo fato do papel machê possuir, principalmente, baixa resistência ao impacto e alta sensibilidade a água, assim, busca-se desenvolver um compósito utilizando o papel como reforço e uma resina biodegradável como matriz. Dessa forma, criou-se um grupo formado pela estudante de Design, Bárbara Zanella, pela doutoranda da Engenharia dos Materiais, Clarissa Angrizani, as doutorandas em Design, Eliana Calegari e Jussara Porto. O objetivo do grupo é desenvolver um material a partir do resíduo do papel que seja resistente mecanicamente, durável e impermeável para ampliar o leque de aplicações de produtos que possam ser feitos a partir do papel.

No que diz respeito aos materiais compósitos, existem diferentes definições, mas na engenharia de materiais refere-se a uma fibra (como material de reforço) e uma matriz (que envolve o material de reforço). O interesse por este tipo de material deve-se ao fato da sua resistência mecânica e dureza específica serem excelentes quando comparado aos materiais tradicionais. Propriedades estas que dependem do tipo, da geometria e orientação do reforço, processamento do compósito e material utilizado como matriz, que pode ser termorrígido ou termoplástico. A matriz tem como função transferir a carga para as fibras, proteger as fibras da abrasão e ao ataque ambiental (MAHAJAN; AHER, 2012). No entanto, os compósitos de origem sintética, em que a fibra geralmente é de vidro ou de carbono e a matriz um polímero termoplástico ou termorrígido, há dificulta na reciclagem ou na reutilização, resultando em problemas de descarte após a sua vida útil (TAIB et al., 2010).

Neste sentido, uma alternativa para amenizar estes problemas é o desenvolvimento de biocompósitos a partir de materiais de fontes renováveis, em que o reforço é constituído por fibras vegetais e a matriz por um polímero biodegradável. Dessa forma, estes materiais podem ser uma solução parcial para os problemas ambientais ocasionados pelo excesso de resíduos sólidos descartados principalmente os de origem polimérica (WU; LIAO, 2012).

Neste contexto, foram realizados experimentos preliminares com o resíduo do papel como reforço para o biocompósito e a resina poliuretana vegetal à base de óleo de mamona por meio da moldagem por compressão. Este processo consiste em transformar um material, depositado na cavidade de um molde, em uma peça de forma definida, através da aplicação de pressão com ou sem aquecimento (CANEVAROLO, 2002). Este é um dos processos muito utilizados para a conformação de compósitos devido ao baixo custo. O experimento resultou em uma placa uniforme com $80 \%$ do resíduo do papel, pode-se constatar visualmente que a matriz aderiu o reforço, como pode ser observado na Figura 5.

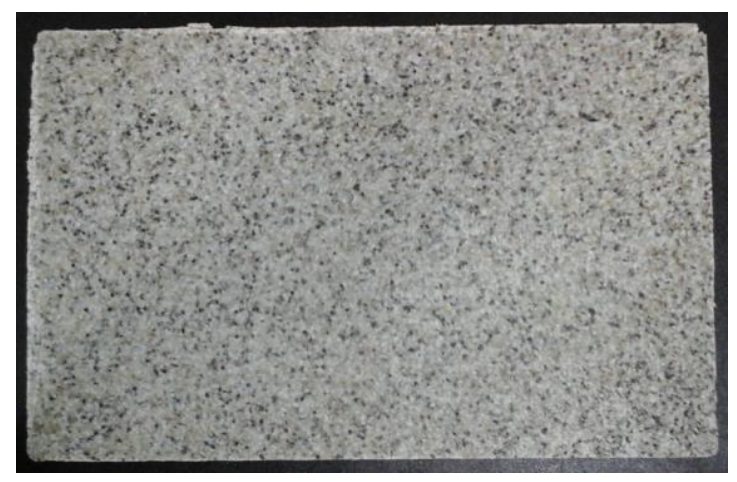

Figura 5 - Biocompósito a base de resíduo de papel e resina poliuretana vegetal de mamona.

Fonte: Elaborado pelos autores, com base na pesquisa realizada. 
A partir deste experimento preliminar, conclui-se que o papel possui potencial para ser utilizado como reforço para biocompósitos. Contudo, a resina poliuretana vegetal à base de mamona, apesar de ser de fonte renovável não é biodegradável, o que não está de acordo com o objetivo da pesquisa do grupo. Dessa forma, o grupo encontra-se realizando novos experimentos com outros tipos de resinas que sejam biodegradáveis e que atendam aos requisitos de conferir boa resistência ao impacto, durabilidade e baixa sensibilidade à umidade.

\section{CONCLUSÃO}

Com a consolidação da pesquisa no Brasil, através do CNPQ, se percebe a ampliação e atualização dos grupos de pesquisa nas instituições de ensino no Brasil, sendo estes grupos, os responsáveis por grande parte das investigações realizadas na atualidade e pela formação de inúmeros pesquisadores. Para o NDePP, é importante ressaltar a evolução que ocorreu através da criação dos grupos de pesquisa, porque os estudos foram sendo desenvolvidos, obtendo-se maior conhecimento em várias áreas; e com isso, vieram outros questionamentos e novas necessidade de pesquisas, fazendo com que se gerassem novos grupos interdisciplinares para novos estudos.

Neste artigo, através dos relatos das pesquisas, pode-se demonstrar a importância do desenvolvimento dos grupos que foram se constituindo ao longo dos anos. Constatou-se também, a importância da interdisciplinaridade na pesquisa, em que novos conhecimentos se somaram para a busca de melhores resultados. Desta forma, colaboradores de outras áreas que agregaram conhecimento, propiciaram o debate e a troca de experiências e promoveram a evolução das pesquisas já existentes.

Por meio deste núcleo, busca-se atingir os níveis de qualidade e excelência que resultem em melhoria ambiental, social e organizacional. Assim, através do objetivo inicial do NUDEP, que tem como foco a criação de produtos com materiais biodegradáveis e sustentáveis, conclui-se que os resultados obtidos até o momento são positivos e o retorno impulsiona o projeto a seguir adiante. Acredita-se que o relato das práticas já implantadas e as em fase de estudo, ajudam a traçar as diretrizes para que possam ser desenvolvidas as ações previstas além das que serão reconhecidas no decorrer do trabalho.

Salienta-se que as constantes pesquisas são extremamente importantes, e observa-se que a pesquisa ainda deve evoluir, mas pela maneira como estão sendo conduzidos os estudos, na qual promovem a interação com a comunidade de pesquisa e a evolução dos conhecimentos; este estudo nos demonstra que o processo com os grupos que estão estabelecidos no NDePP, é a forma do aperfeiçoamento das ações e de direcionamentos futuros para a realização dos objetivos.

A importância destas pesquisas, tanto para a divulgação do trabalho dos grupos, quanto para o meio acadêmico e para a sociedade, se consolida na medida em que se promovam mudanças significativas para o meio ambiente e se colabore com o bem estar social. Desta forma, pode vir a servir como modelo a ser replicado por outras instituições, tornando-se referência na área.

Com a elaboração deste artigo, buscou-se a divulgação das práticas de pesquisas desenvolvidas no NDePP à comunidade externa, discentes e docentes, para que possa ocorrer a expansão do projeto e a evolução do trabalho de pesquisa, na qual se pretende ainda, a busca de diferentes materiais compósitos, a estruturação dos grupos de pesquisa, a organização das vertentes de pesquisa, o envolvimento com outros laboratórios; para viabilidade e continuidade do desenvolvimento de outros produtos.

\section{REFERÊNCIAS}

CALEGARI, E. P. Estudo da aplicação de compósitos biodegradáveis à base de biopolímero e fibras de curauá no design de produto. Dissertação de Mestrado. Programa de Pós-Graduação em Design. UFRGS. Porto Alegre. 2013

CANEVAROLO, J. S. V. Técnicas de caracterização em polímeros. São Paulo: Artliber, 2004.

CENTRO SEBRAE DE SUSTENTABILIDADE. Gestão Sustentável nas Empresas. . Acesso em 25 de julho de 2015.

CURTIS, M. do C. Núcleo de Desenvolvimento de Produtos em Papel: uma retrospectiva para Ação. Arquivos da Gráfica da UFRGS. 2011. 
DEMAJOROVIC, J. Sociedade de risco e responsabilidade socioambiental: perspectivas para a educação corporativa. 2000. 254p. Tese - Doutorado - Faculdade de Educação, Universidade de São Paulo, São Paulo, 2000.

KAZAZIAN, T. Haverá a idade das coisas leves: design e desenvolvimento sustentável. São Paulo. Editora Senac são Paulo, 2005.

MAHAJAN, G.V.; AHER, V. S. Composite Material: A Review over Current Development and Automotive Application. International Journal of Scientific and Research Publications, Volume 2, Issue 11, 2012.

PICCOLI, M.; MEDITSCH, A. de O.; PORTO, J. Estudo experimental sobre o tingimento natural do papel machê. In: Anais 3o Fórum Internacional Ecoinovar, 2014, Santa Maria.

PORTO, J. Avaliação do uso da metodologia Human Centered Design (HCD) para qualificação de técnicas de produção de artefatos a partir de resíduos de papel em comunidade de Porto Alegre/RS. 2013. 139p. Dissertação - Programa de Pós-Graduação em Design, Centro Universitário Ritter dos Reis, Porto Alegre, 2013.

PRODANOV, C. C.; FREITAS, E. C. de. Metodologia do trabalho científico: métodos e técnicas de pesquisa e do trabalho acadêmico. Novo Hamburgo: Ed. Feevale, 2009.

SANTOS, J. V. T.; STEIL, C. (org.). Introdução. In: Humanas: Revista do Instituto de Filosofia e Ciências Humanas - Projetos Sociais. Universidade Federal do Rio Grande do Sul, Instituto de Filosofia e Ciências Humanas. Vol.24, n¹/2, Porto Alegre, 2001.

TAIB, R. M.; RAMARAD, S.; ISHAK, Z. A. M.; TODO, M. Properties of Kenaf Fiber/Polylactic Acid Biocomposites Plasticized with Polyethylene Glycol. Polymer composites, 2010.

UFRGS. 2012. Material de Apoio Secretaria de Gestão Ambiental. Disponível em: <http://www.ufrgs.br/sga/biblioteca-sga/material-de-apoio-sga>. Acesso em: 08 jul. 2013.

UFRGS. 2013. Gráfica da UFRGS. Disponível em: <http://www.ufrgs.br/graficaufrgs>. Acesso em 06 jul. 2015.

WU, C. S.; LIAO, H. T. Polycaprolactone-Based Green Renewable Ecocomposites Made from Rice Straw Fiber: Characterization and Assessment of Mechanical and Thermal Properties. Industrial \& Engineering Chemistry Research, 51, p. 3329 - 3337, 2012. 